\title{
Complete and assembled genome sequence of an NDM-5 and CTX-M-15 producing Escherichia coli sequence type 617 isolated from wastewater in Switzerland
}

\author{
Zurfluh, Katrin ; Stevens, Marc J A ; Stephan, Roger ; Nüesch-Inderbinen, Magdalena
}

\begin{abstract}
OBJECTIVES: Carbapenem-resistant Escherichia coli have emerged worldwide and represent a major challenge to effective healthcare management. Here we report the genome sequence of an NDM-5- and CTX-M-15-producing E. coli belonging to sequence type 617 isolated from wastewater treatment plant effluent in Switzerland. METHODS: Whole-genome sequencing of E. coli 657SK2 was performed using Pacific Biosciences (PacBio) single-molecule real-time (SMRT) technology RS2 reads (C4/P6 chemistry). De novo assembly was carried out using Canu 1.6, and sequences were annotated using the NCBI Prokaryotic Genome Annotation Pipeline (PGAP). RESULTS: The genome of E. coli 657SK2 consists of a 4.9-Mbp chromosome containing blaCTX-M-15, genes associated with virulence [fyuA, hlyE, the pyelonephritis-associated pili (pap) gene cluster and the yad gene cluster], the copper resistance gene pco, and genes associated with resistance to quaternary ammonium compound (QAC) disinfectants (emrA, mdfA and sugE). A 173.9-kb multidrug resistance IncFII-FIA-FIB plasmid was detected harbouring aadA2, aadA5, blaNDM-5, blaOXA-1, cat, drfA, drfA17, the mph(A)-mrx-mphR cluster, the tetA-tetC-tetR cluster, and the virulence genes iutA and ylpA. CONCLUSIONS: The genome sequence of E. coli $657 \mathrm{SK} 2$ provides information on resistance mechanisms and virulence characteristics of pathogenic E. coli harbouring blaNDM-5 and blaCTX-M-15 that are spreading into the environment via urban wastewater.
\end{abstract}

DOI: https://doi.org/10.1016/j.jgar.2018.08.015

Posted at the Zurich Open Repository and Archive, University of Zurich

ZORA URL: https://doi.org/10.5167/uzh-167647

Journal Article

Accepted Version

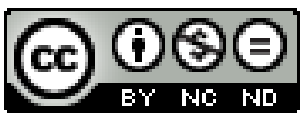

The following work is licensed under a Creative Commons: Attribution-NonCommercial-NoDerivatives 4.0 International (CC BY-NC-ND 4.0) License.

Originally published at:

Zurfluh, Katrin; Stevens, Marc J A; Stephan, Roger; Nüesch-Inderbinen, Magdalena (2018). Complete and assembled genome sequence of an NDM-5 and CTX-M-15 producing Escherichia coli sequence type 617 isolated from wastewater in Switzerland. Journal of Global Antimicrobial Resistance, 15:105-106. DOI: https://doi.org/10.1016/j.jgar.2018.08.015 
1 Complete and assembled genome sequence of an NDM-5 and CTX-M-15 producing

2 Escherichia coli sequence type 617 isolated from wastewater in Switzerland

3

4 Katrin Zurfluh ${ }^{1}$, Marc J.A. Stevens ${ }^{1}$, Roger Stephan ${ }^{1 *}$ Magdalena Nüesch-Inderbinen ${ }^{1}$

5

6

$7{ }^{1}$ Institute for Food Safety and Hygiene, Vetsuisse Faculty, University of Zurich, Zurich,

8 Switzerland

9

10

11

12

13

$14 *$ Corresponding author:

15 Roger Stephan, Institute for Food Safety and Hygiene, Vetsuisse Faculty, University of

16 Zurich, Winterthurerstrasse 272, CH-8057 Zurich, Switzerland.

17 Phone +41 4463586 51, Fax +41 4463589 08, e-mail stephanr@fsafety.uzh.ch

18 


\section{Abstract}

20 Objectives: Carbapenem-resistant Escherichia coli have emerged worldwide and represent a

21 major challenge to effective health care management. Here, we report the genome sequence 22 of an NDM-5 and CTX-M-15 producing E. coli belonging to sequence type 617, isolated 23 from wastewater treatment plant effluent in Switzerland.

24 Methods: Whole genome sequencing of E. coli 657SK2 was performed using Pacific 25 Biosciences (PacBio) single-molecule real-time (SMRT) technology RS2 reads (C4/P6 26 chemistry). De novo assembly was carried out using CANU 1.6, and sequences were 27 annotated using the NCBI Prokaryotic Genome Annotation Pipeline.

28 Results: The genome of E. coli $657 \mathrm{SK} 2$ consists of a 4.9-Mbp chromosome containing $b l a_{\mathrm{CTX}-\mathrm{M}-15}$, genes associated with virulence $(f y u A, h l y E$, the pyelonephritis-associated pili (pap) gene cluster, and the yad gene cluster), the copper resistance gene $p c o$, and genes associated with resistance to quaternary ammonium compound (QAC) disinfectants, emrA, $m d f A$, and $s u g E$. A 173,9-kb multidrug resistance IncFII-FIA-FIB plasmid was detected harbouring aadA2, aadA5, bla $\mathrm{NDM}_{-5}$, bla $\mathrm{OXA}_{\mathrm{O}-1}$, cat, $\operatorname{drf} A, \operatorname{drfA17}$, the $m p h(A)-m r x-m p h R$ cluster, the tetA-tetC-tetR cluster, and the virulence genes $i u t A$, and $y \operatorname{lp} A$.

Conclusions: The genome sequence of $E$. coli $657 \mathrm{SK} 2$ provides information on resistance mechanisms and virulence characteristics of pathogenic E. coli harbouring bla $a_{\mathrm{NDM}-5}$ and bla $a_{\text {СТХ-M-15 }}$ that are spreading into the environment via urban wastewater.

\section{Keywords}


43 The dissemination of carbapenemase-producing Enterobacteriaceae (CRE) constitutes a

44 challenge to effective healthcare management worldwide. New Delhi metallo- $\beta$-lactamases (NDM) positive E. coli have been identified in infected or colonised patients in the clinical setting and sporadically from animal or environmental sources [1]. The NDM-5 variant, which is infrequently detected worldwide, differs from NDM-1 by enhanced hydrolytic activity against carbapenems and the point mutations at positions 88 (Val $\rightarrow$ Leu) and position 154 (Met $\rightarrow$ Leu).

E. coli $657 \mathrm{SK} 2$ was isolated from wastewater treatment plant effluent which is released into the river Rhine near Basel, Switzerland in December 2015 [2]. DNA extraction was performed with the Wizard ${ }^{\circledR}$ Genomic DNAPurification Kit according to the manufacturers protocol (Promega AG, Dübendorf, Switzerland). The genome was sequenced at the Functional Genomics Center Zurich (FGCZ), Switzerland, using two single-molecule realtime (SMRT) cells on a PacBio RS II (Pacific Biosciences, Menlo Park, CA, USA). The raw reads were filtered using the RS Filter Only protocol in the SMRT-portal (Pacific Biosciences) using standard settings. A total of 84,248 reads with an average length of 10,450 bp were selected, corresponding to $880,442,668$ sequenced basepairs and a genome coverage of approximately 175 fold. The reads were assembled using Canu 1.6 [3] with the option "pacbio-filtered" and an estimated genome size of 5.0 Mbp. The Canu output consisted of 4 contigs which were further polished in CLC workbench 7 (CLC, Aarhus, Denmark), eventually resulting in one chromosome, one phage and one plasmid encoding contig. The chromosomal origin of replication was identified using DoriC 5.0 and plasmidal origin of replication were determined by PlasmidFinder 1.3. The start of the chromosome was set $9 \mathrm{bp}$ upstream of the first DnaA box in the origin of replication region. The genome was annotated by the NCBI Prokaryotic Genomes Automatic Annotation Pipeline (GPAP) server. 
The chromosome of $E$. coli $\mathrm{ST} 617657 \mathrm{SK} 2$ has a $\mathrm{G}+\mathrm{C}$ content of $49.9 \%$ and consists of a 4,889,056-bp chromosome. One extra-chromosomal element of 173,883 bp was identified and designated p657SK2. The Prokaryote Genome Annotation Pipeline (PGAP) at NCBI predicted 5250 genes and 5125 protein-coding sequences. A total of 296 frame-shifted genes were identified by PGAP and manual analysis revealed that 128 frame shifts were caused by homopolymer sequencing errors. These 128 frameshifts were corrected using the genome of E. coli $\mathrm{K} 12$ substrain MG1655 (accession number CP027060.1) as leading sequence.

The chromosome of $E$. coli $657 \mathrm{SK} 2$ contains the $\beta$-lactam resistance gene bla $a_{\mathrm{CTX}-\mathrm{M}-15 \text {, }}$ virulence associated genes $f y u A, h l y E$, the pyelonephritis-associated pili (pap) gene cluster, and the yad gene cluster, as well as the copper resistance gene pco, and genes associated with resistance to quaternary ammonium compound (QAC) disinfectants, emrA, $m d f A$, and sugE. Plasmid p657SK2 is a multireplicon IncFII-FIA-FIB plasmid assigned to the FAB formula F31:A4:B1 by the IncF replicon typing scheme (http://cge.cbs.dtu.dk/services/pMLST/) [4]. In addition, the presence of a second FII allele (F36) was observed, which is composed of repA2, герA6 and truncated repA1. Plasmid p657SK is a multidrug resistance (MDR) plasmid encoding the following resistance genes: aminoglycosides (aadA2 and aacA5), $\beta$ lactams (bla $a_{\mathrm{NDM}-5}$, bla $\left.a_{\mathrm{OXA}-1}\right)$, bleomycin $($ ble $)$, chloramphenicol (cat), macrolides $(m p h(A)-$ $m r x$-mphR cluster), tetracycline (tet $A$-tet $C$-tet $R$ cluster), trimethoprim ( $\operatorname{drf} A$ and $\operatorname{drfA17})$. The plasmid further contains virulence genes iutA, $y \operatorname{lp} A$, and two truncated copies of the QAC resistance gene emrE.

Genes for four toxin/antitoxin systems were detected $(c c d A / c c d B$, hok, pemI/pemK, and $\operatorname{vapB} / \operatorname{vap} C)$.

Plasmid p657SK2 closely resembles the bla $a_{\mathrm{CTX}-\mathrm{M}-15}$ encoding plasmids pCA14 (CP009231) and pCA28 (P009232) from E. coli causing community-acquired infections in the USA [5], each with $85 \%$ coverage and $99 \%$ identity. The bla $a_{\mathrm{NDM}-5}$ gene is located downstream of 
93

94

95

96

97

98

99

100

101

102

103

104

105

106

107

108

109

110

111

112

\section{$113 \quad$ Funding}

114 This work was supported by funding from the University of Zurich.

(Figure 1). The upstream region of the bla $a_{\mathrm{NDM}-5}$ gene contains a resistance gene locus consisting of IS6100 and the $m p h(A)-m r x-m p h R$ cluster. carbapenemase gene to disseminate to other plasmids and species. poses a threat to human and animal health. version of this genome.

truncated ISAba125 and upstream of ble. The genetic environment of this highly conserved arrangement is on a $22.7 \mathrm{kbp}$ region which is bracketed by two IS26 elements and constitutes a putative composite transposon containing a class I integron, aminoglycoside resistance genes $a a d A 2$ and $a a c A 5, d r f A, d r f A 17$, and sull, and an insertion sequence common region 1 (ISCR1) replicase (Figure 1). The intI1 gene downstream of bla $a_{\mathrm{NDM}-5}$ is truncated by IS26

The occurrence of bla NDM-5 $_{5}$ in this genetic constellation suggests a high potential of this

In summary, we report the draft genome of a carbapenem resistant E. coli ST617 isolated from wastewater treatment plant effluent. The occurrence of NDM-5 producing E. coli in treated urban wastewater that is released into one of the European continent's major rivers is cause for concern. Environmental pollution with multidrug resistant, NDM-producing E. coli harbouring genes associated with virulence and resistance to heavy metals and disinfectants

Sequence and annotation data of the genome have been deposited at GenBank under accession numbers $\underline{\mathbf{C P 0 2 7 7 0 1}}$ (chromosome), and $\underline{\mathbf{C P 0 2 7 7 0 3}}$ (p657SK2). This is the first

\section{Competing interests}

117 None declared. 
118

119 Ethical approval

120 Not required.

121 
124 [1] Johnson AP, Woodford N. Global spread of antibiotic resistance: the example of New 125 Delhi metallo- $\beta$-lactamase (NDM)-mediated carbapenem resistance. J Med Microbiol $126 \quad 2013 ; 62: 499-513$.

127 [2] Zurfluh K, Bagutti C, Brodmann P, Alt M, Schulze J, Fanning S et al. Wastewater is a reservoir for clinically relevant carbapenemase- and 16s rRNA methylase-producing Enterobacteriaceae. Int J Antimicrob Agents 2017; 50:436-440.

130 [3] Koren S, Walenz BP, Berlin K, Miller JR, Bergman NH, Phillippy AM. Canu: scalable and accurate long-read assembly via adaptive k-mer weighting and repeat separation. Genome Res 2017; 27:722-736.

[4] Carattoli A, Zankari E, Garcìa-Fernandez A et al. PlasmidFinder and pMLST: in silico detection and typing of plasmids. Antimicrob Agents Chemother 2014; 58:3895-3903.

[5] Li JJ, Spychala CN, Hu F, Sheng JF, Doi Y. Complete nucleotide sequences of bla $a_{\mathrm{CTX}-\mathrm{M}^{-}}$ harboring IncF plasmids from community-associated Escherichia coli strains in the United States. Antimicrob Agents Chemother 2015; 59:3002-3007. 
$139 \quad$ Figure legend

140

141 Linear map of the $22.7 \mathrm{kbp}$ region of p657SK2 containing bla $a_{\mathrm{NDM}-5}$. Antimicrobial resistance

142 genes are coloured in red, insertion sequences (IS) are shown in dark or light blue, resistance

143 to quaternary ammonium compound (QAC) disinfectants are shown in green, other genes are

144 shown in burgundy.

$145 a a c A 5$, aminoglycoside $\mathrm{N}\left(6^{\prime}\right)$-acetyltransferase gene; bla $a_{\mathrm{NDM}-5}$, gene coding for the New

146 Delhi metallo-ß-lactamase; ble, bleomycin resistance gene; $d f r A$, dihydrofolate reductase

147 gene; $\operatorname{dos} P$, gene for oxygen sensor protein; $d s b C$; disulfide bond isomerase II gene; $\Delta e m r E$,

148 truncated quaternary ammonium compound-resistance gene; intI1, integron integrase gene;

149 ISCR-1, gene for the rolling circle replicase of the insertion sequence common region 1

150 transposase; $\operatorname{srp} C$, gene for chromate transport protein; sull, sulfonamide resistance gene;

$151 \operatorname{trp} F$, phosphoribosylanthranilate isomerase gene. 